\title{
Crossing the Symbolic Threshold
}

Author's postprint of: "Crossing the symbolic threshold: a critical review of Terrence Deacon's The Symbolic Species", Philosophical Psychology, VOL. 15, NO. 2, 2002

Dr. David Lumsden

Department of Philosophy and Religious Studies

University of Waikato

Private Bag 3105

Hamilton 3240

New Zealand

phone: +6478384466 Ext. 6574

fax: +64 78384018

email: d.lumsden@waikato.ac.nz

\begin{abstract}
Terrence Deacon's views about the origin of language are based on a particular notion of a symbol. While the notion is derived from Peirce's semiotics, it diverges from that source and needs to be investigated on its own terms in order to evaluate the idea that the human species has crossed the symbolic threshold. Deacon's view is defended from the view that symbols in the animal world are widespread and from the extreme connectionist view that they are not even to be found in humans. Deacon's treatment of symbols involves a form of holism, as a symbol needs to be part of a system of symbols. He also appears to take a realist view of symbols. That combination of holism and realism makes the threshold a sharp threshold, which makes it hard to explain how the threshold was crossed. This difficulty is overcome if we take a mild realist position towards symbols, in the style of Dennett. Mild realism allows intermediate stages in the crossing but does not undermine Deacon's claim that the threshold is difficult to cross or the claim that it needs to be crossed quickly.
\end{abstract}




\section{Deacon's account of the origin of language}

In The Symbolic Species, Terrence Deacon (1997) has various theses about the origin of language linked to the central idea that what is crucial is the development of the human capacity for symbolic thought. One of the things that he sets out to explain is the absence of simple languages (p. 12). Deacon's work can be seen as part of a new wave of interest in the origin of language, which encompasses investigation of the social setting for language development, and the biological basis of various features of language (Hurford et al., 1998). This paper deals especially with the philosophical bases of Deacon's views.

\subsection{The Symbolic Threshold}

The primary title of Deacon's book identifies humans as the symbolic species. This is not merely the claim that humans employ those symbols that constitute human natural languages, but also the claim that the underlying human cognitive structure is symbolic in nature (p. 22). This puts the cognitive aspect of symbolic thinking at centre stage in the account of the origin of language rather than emphasizing complex syntax, as Chomsky (1988) does.

While some talk of symbols focuses on their physical form, Deacon's focus is on the ability of symbols to represent things. Briefly, he considers that cognitive symbols must form part of a symbol system and their representative function depends crucially on their relationships with other symbols in the system (p. 83). More will be said about that notion of a symbol in section 2. Deacon's notion of a symbolic threshold refers to a moment in evolutionary development before which creatures do not employ symbolic reference. The existence of a threshold is related to the view that symbols need to be part of a symbolic system. Crossing that threshold is difficult and he speaks of a 'symbolic barrier' (p. 44). He dates the transition to the time of the late Australopithecines (p. 348), though allows that other primates with special training can make that leap (p. $84 \mathrm{ff})$. 
In Deacon's view, it is necessary to have crossed the symbolic threshold for there to be word meaning and reference. Thus, linguistic meaning is linked to symbolic thought, which suggests an internalist account of linguistic meaning. On the other hand, the social character of meaning is also recognized. The social character is said to release the meaning of words from dependence on merely personal experience ( $\mathrm{p}$. 450). Nonetheless, once acquired, language can be employed in internal cogitation.

While this paper focuses primarily on the central notion of crossing a symbolic threshold, it will be useful to look at the broader context in which that idea is developed

\subsection{Co-evolution}

The subtitle of Deacon's book is, "The co-evolution of language and the brain." The notion of co-evolution is well established in biology, describing, for example, how certain species of flowering plants evolved reciprocally with certain species of insect pollinators, so that each adapted to suit the characteristics of the other. Our interest is in the way the biological evolution of the brain and the social evolution of language involved that mutual adaptation. A modification to the brain that supports language better enables language to flourish and improves the fitness of individuals. Deacon (p. 413) says the species has now reached the point where the capacity to acquire language is over-engineered so as to be fail-safe. On the other side, a modification to a particular language that allows it to be learned more efficiently and at an early age will help that particular language to flourish at the expense of others. In this way, language, like a parasite, adapts itself to its host as well as the host adapting itself to the parasite.

The social evolution of language happens a lot faster than the biological evolution of the brain and there is scope for language to do a lot of adapting. Deacon (p. $137 \mathrm{ff}$ ) argues that the immature brain has characteristics that enhance the ability to acquire symbolic thought, which channels the social evolution of language to favour early acquisition. Deacon believes that work by Chomsky and his followers into innate 
language structures, which is offered as an explanation of how language is learnable, neglects the way that language adapts to foster its learnability (Deacon, 1997, p. 102 ff).

\subsection{Brain Development}

The crossing of the symbolic threshold in evolution, as Deacon describes it, was based, in part, on a development in brain structure. An increase in brain size is one notable feature of the evolution of pre-humans into humans, but overall brain size is not the major issue. The striking difference over this period was expansion of the cerebral cortex, which distorts the overall pattern of neural connections. The prefrontal cortex of a human is about twice the size that an ape of our size would have (p. 218). Deacon links this explicitly with crossing the symbolic threshold.

Deacon (pp. 264-7, 300) claims the pre-frontal cortex, which supports higher level associations, can support the creation of a system of virtual symbols, not purely based in the pre-frontal cortex, but distributed in various parts of the brain. He discusses extensively the role of the prefrontal cortex in relation to other parts of the human brain, including the cerebellum, and discusses experimental evidence connecting the prefrontal cortex with different forms of behavior. For example, he shows how it is linked with behavior being removed from immediate stimulus control (pp. 259-264), which is consistent with his notion of a symbol.

\subsection{The Social Circumstances}

Deacon (pp. 384-401) also has a thesis about the social circumstances in which this kind of symbol processing, which carries processing overheads, was able to emerge. Those circumstances involved group hunting, which provided protein important for survival, especially for mothers and children. Males, who had exclusive sexual access to certain females and thereby avoided supporting other men's children, tended to have more descendants and so behavior enforcing that exclusivity became entrenched. But the group living created many opportunities for promiscuity, which led to 
instability. The solution was the introduction of natural language, which can allow for promises of exclusivity to be made and enforced by the community.

This is a highly speculative account and would probably attract Lewontin's (1996, p. 108) dismissive description as merely a story. Whether or not this particular part of Deacon's account is plausible, there need to be some kinds of evolutionary benefits accruing from language acquisition that provide a plausible evolutionary path to our current level of adaptation to language. Deacon (p. 352) notes that once symbolic communication supported one social function it was available to support many others.

First steps in an evolutionary, or indeed co-evolutionary, process are particularly fragile. At the stage where the brain is still not well adapted to symbol processing, early use of language is hard for individuals to acquire. Deacon (pp. 401-3) has an explanation of how that difficulty could be overcome. He suggests that in rituals there can be frequent repetition of a series of signs, which are removed from their objects. This occurs in a situation of emotional intensity where the signs can be seen in a new light. His thought is that the relationships of signs to each other, which he sees as central to being a symbol, can be appreciated in those circumstances. As will be discussed in section 4.2, Deacon may need to view ritual as a structure that strictly preceded language. Again, this scenario is speculative, but does further explore possible paths in the evolutionary process.

\section{Deacon's notion of a symbol}

We need to scrutinize Deacon's notion of a symbol. He initially identifies the special nature of human natural language, in contrast to other animal communication systems, on the basis of its combinatorial power (p. 32). But the key difference is humanity's possession of a mental symbol system. We need to get as clear as we can about that notion of a symbol. 


\subsection{The basis in Peirce}

Deacon derives his notion of a symbol from Peirce. One of three tripartite distinctions made by Peirce in his semiotics is the distinction between icon, index and symbol (Buchler, 1955, pp. 98-119). An icon refers by way of resemblance, as a picture resembles its object. An index refers by way of being really affected by its object. For example, a weathercock, is an index of the direction of the wind. The mind is drawn by the index to think of the object. A symbol is something general that is based on law, which is explained as involving convention or habit. Note that Peirce's notion of habit can include something inborn (Buchler, 1955, p. 113). Common nouns are given as examples of symbols, as are signs of propositions and arguments.

Deacon's view is that while other animals employ icons and indices, essentially only humans have crossed the symbolic threshold and can employ symbols. The symbols are supposed to rest on a base of icons and indices and are taken to be some form of abstraction from the indices (p. $79 \mathrm{ff}$ ). What is said to be crucial is the way that symbols relate to other symbols, as will be discussed in the next section.

There are reasons to doubt whether Peirce's semiotics really provides the appropriate philosophical background for Deacon's claims. While Peirce did believe in a hierarchical relationship among icon, index and symbol, his analysis intertwined those notions in ways that are at odds with the sort of discrete stages that Deacon needs.

In Peirce's scheme, an algebraic formula is iconic, for it can reveal unexpected truths, as a map can (Buchler, 1955, pp. 105-6). Quantifiers are identified as indefinite indices (p. 111). In neither case do we have something more primitive than human language, but rather something sophisticated. Also, while an ordinary proposition is in itself a kind of symbol, its interpretant must be taken as a form of index (p. 111), pointing to what Hookway (1985, p.138) describes as 'a state of affairs'. (It is a general feature of Peirce's system that any sign requires an interpretant: another sign that provides an interpretation of the first.) For Peirce, icon, index and symbol are all essential characteristics of a scientific language. 
Peirce describes demonstrative and personal pronouns as indices (Buchler, 1955, p. 107), although he also employs non-linguistic examples such as the weathercock. Linguistic indices (or indexicals, as we now say) should be regarded as sophisticated rather than primitive kinds of signs, as shown by their comparatively late place in child language acquisition. The massive indexicality of a sentence such as "I am here now," is not evidence of it being a more primitive utterance that could express a more primitive thought available to less advanced minds. Elizabeth Bates (1990, p. 169) points out that young children avoid the problem of dealing with indexicals by using their names to refer to themselves, employing the 'Tarzan-Jane' strategy.

In David Kaplan's (1989, section VI) terminology, indexicals have a variable 'character'; that is their 'content' varies systematically from context of utterance to context of utterance. Or, as Elizabeth Bates (1990, p. 166) puts it, an indexical like 'I' involves shifting reference. A linguistic indexical thus involves a particular kind of sophisticated convention and is strikingly different from a sign that an animal can be trained to associate with a particular object or behavior. Indexicals are an integral and fully fledged part of the symbolic system that is language.

Peirce himself was able to describe the special nature of a linguistic indexical. He describes a demonstrative pronoun, which is a form of indexical, as a 'rhematic indexical legisign' (Buchler, 1955, p. 116). 'Rhematic' just means that it represents a certain kind of possible object (Buchler, 1955, p. 103) while 'legisign' refers to some kind of conventional sign. Thus Peirce at least has a way of describing the conventionality of linguistic indexicals using his more elaborate scheme. But Deacon merely employs the icon/index/symbol hierarchy and sees the move to symbol use as a fundamental cognitive shift. For him, an index is essentially more primitive than a symbol. Deacon (p. 86) considers that when, for example, a chimp is taught to associate an arbitrary shape with bananas (to be discussed in the next section) the relationship is indexical. That kind of case does not appear to fit clearly into the pattern that Peirce describes. It is unlike the case of the weathercock in which the orientation of the sign is directly affected by the thing it represents. In Deacon's hands, 'index' thus tends to cover any sign individually connected with the world, 
which is at variance with Peirce's notion. In the next section we shall see how indices work for Deacon as the preparatory stage for ascending to the level of symbols.

If Deacon is to account for the sophisticated nature of linguistic indexicals he needs to hold that they are essentially symbolic. But Deacon (p. 80) says that, "Words can serve indexical functions as well, and are sometimes used for this purpose almost exclusively, with minimal symbolic content." He includes 'there' as one of his examples. While he does allow that there is some symbolic content, he appears to be overlooking the sophistication of the use of indexical words in language.

Thus, Deacon's view on language and thought should be separated from Peirce's semiotics, as Deacon's application is not wholly consistent with Peirce's own notions. Also, the way Peirce's distinction between index and symbol gets reflected in Deacon's work is in danger of distorting our appreciation of the role of linguistic indexicals. We would be wise to address Deacon's notion of a symbol on its own terms and not assume that its origin in Peirce's work on semiotics makes it perfectly clear.

\subsection{Deacon's notion of a symbol without Peirce}

Leaving Peircean semiotics behind, here are some central features of Deacon's notion of a symbol.

Firstly, a symbol's connection with its reference is not sustained by repeated association between a symbol and an object or behavior (Deacon, 1997, pp. 67-8). Behaviorist techniques can be used to teach an intelligent mammal to employ arbitrary signs that are linked with specific objects or behaviors. Without continual renewal of that correlation, the use of the signs lapses. Symbols, in contrast, can be employed with little or no correlations with the behavior or object.

Secondly, symbols achieve relative independence from those correlations by forming a system of symbols. Thus they have important connections with other symbols, which compensates for a less robust correlation in experience with aspects of reality. 
"Their indexical power is distributed, so to speak, in the relationships between words" (p. 83). This distribution can certainly be recognized in the way that words have their meaning linked by definition to other words. So a word does not need to be correlated with reality all by itself.

Deacon stresses that the distribution of indexical power in a system of symbols depends crucially on syntactic relationships. He says, “... syntactic structure is an integral feature of symbolic reference" (p. 100). He also says, "Symbolic reference derives from combinatorial possibilities and impossibilities, and we therefore depend on combinations both to discover it (during learning) and to make use of it (during communication)" (p. 83). This feature of symbols appears to be a central part of Deacon's attempt to explain the 'miracle of word meaning and reference' (p. 43.

Deacon (p. 84 ff) throws further light on his understanding of the way symbols form a system when he discusses how the chimps, Sherman and Austin, allegedly cross the symbolic threshold. (Savage-Rumbaugh et al., 1978). They are taught a language consisting of arbitrary shapes, called 'lexigrams', which correspond to whole words in a natural language. The chimps can utter sentences by depressing special keys on a computer keyboard, which are marked with those lexigrams. They had been trained to avoid ungrammatical sentences of the language, which had a simple verb-noun structure.

Initially there were only 2 verb lexigrams, one of which is described as 'give' but refers to the process of supplying solid food by a dispenser. There were 4 noun lexigrams which referred to drink and to solid food. As new lexigrams were taught they were picked up quickly and fitted into the structure already learned. Deacon (pp. 89-92) claims that producing the grammatical categories by abstraction saves a significant memory load, and also makes it easier to learn which sentences containing new lexigrams are permissible. There certainly is some potential for economy, particularly when more lexigrams are involved. Rather than have to learn each pair as a permissible sentence it is only necessary to learn which group that lexigram belongs to and then fit it in to the verb-noun structure that is learned. 
On the basis of Sherman and Austin's achievements Deacon (p. 86) claims that "They had discovered that the relationship that a lexigram has to an object is a function of the relationship it has to other lexigrams ..." That insight is described as a process of perceptual recognition of the 'implicit pattern that might be recognized in the relationships between the indexical associations' (p. 93). The insight is supposed to be essentially immediate, taking only the time appropriate to perceptual recognition.

The third feature of symbols that I am extracting from Deacon is that the system of symbols needs to be based on processes that are 'actively and spontaneously adaptive', processes that Deacon speaks of as iconic and indexical. The system must "continuously evolve new means of fitting with and anticipating its environment" (p. 455). This remark comes in the context of what would be required to build an artificial intelligence. For our purposes here, we should note that Deacon's symbolic system needs to be based on the kind of rich, perception based, interactions with the environment that are characteristic of animals.

The final feature of symbols, as mentioned in section 1.4, is that symbols must be sufficient to express promises. Note especially that promises involve a content that concerns something not immediately available, as it relates to the future.

Here in summary, are the points about symbols that I have extracted from Deacon. First, the use and significance of symbols are not merely sustained by frequent correlations with experience. Second, the symbols crucially depend on their place in a system of symbols, which involves both syntactic and semantic relationships among symbols. This is the central feature, which enters into the account of how the leap into symbolic representation takes place. Third, the symbolic system rests on the rich interactions with the environment characteristic of animal brains. Fourth, the symbolic system must allow promises about the future to be made.

\subsection{Simple Languages and the Threshold}

Remember that Deacon's account is supposed to explain why there are no simple languages. Other species have enough intelligence to handle a simple version of a 
combinatorial language and engage in complex communicative behaviors (p. 42). But crossing the symbolic threshold is not easily done. Deacon (p. 378) explains that symbolic learning is in conflict with associative learning and so the early stages would be unstable. The shift into this style of representation is very demanding. "To learn a first symbolic relationship requires holding a lot of associations in mind at once while at the same time mentally sampling the potential combinatorial patterns hidden in their higher order relationships" (p. 93). Only once the benefits of the new form of communication were more fully realized would the benefits really outweigh the costs.

Deacon (p. 44) allows that once there were once simple languages, at the time of humans crossing the symbolic threshold. As brains evolved to cope with them, the languages became more complex and supplanted the simpler versions (p. 45). The difficulty of crossing the threshold means that other species have not been able to make a start in that co-evolutionary process, and hence there are no simple languages to observe currently.

Let us accept that there is a plausible co-evolutionary path from the crossing of the threshold to the present day. It is the period of the crossing of the threshold and the events leading up to it that are difficult to describe. Deacon cannot be intending the notion of co-evolution to apply to that period. Remember that the existence of a threshold is bound up with the way that a symbol must be part of a symbol system, the second feature discussed above. Deacon (p. 378) says, "Only after a complete group (in a logical sense) of interdefined symbols is assembled can any one be used symbolically." This suggests a perfectly sharp threshold. Deacon's very notion of a symbol thus appears to make it hard for him to describe the process of crossing the threshold. We are denied the notion of something that is nearly a symbol, which is what we may need to describe the process leading up to the crossing of the threshold.

In section 4 we will explore a reinterpretation of Deacon's view of symbols that better accommodates the crossing of the threshold. 


\section{Is there a threshold to cross?}

The claim that there is a threshold, which humans have crossed, can be divided into two parts. The first is that simpler animals do not manipulate symbols and the second is that human beings do. Both parts are contentious. For the first part I will consider the case of insects and argue against the claim that they manipulate symbols. For the second part I will compare the symbol manipulation hypothesis with a connectionist hypothesis.

\subsection{Maybe both insects and humans manipulate symbols}

There is a tendency in cognitive science to assume many creatures employ symbolic representation. Charles Gallistel (1998) uses a range of experimental evidence to suggest that various insects have cognitive maps and claims that this involves symbolic representation. If these insects have cognitive maps, then similar considerations are likely to apply to a variety of mammals, birds and reptiles. Here is an example of the relevant sort of accomplishment. An insect is traveling from A to B, is captured and transported to $\mathrm{C}$ and can then establish a more or less direct route from C to B. Certainly that suggests there is some kind of an internal map.

Gallistel (1998, pp. 6-10) argues that in order to do this kind of thing the organism needs to evaluate mathematical functions. These functions can be embedded in each other so that the value of a contained one becomes an argument for the containing one. This makes the computational system 'compositional' in that the component functions have a consistent interpretation. Natural languages are also said to have the property of compositionality in that component words and phrases have a consistent significance in an indefinitely large range of complex sentences. Deacon would have to deny that navigation by a cognitive map is a case of a symbolic process, as he wishes to draw a line between humans and most other creatures. Do cognitive maps fail to meet the characteristics of symbols I have drawn from Deacon?

The first characteristic is that symbols are supposed not to be dependent on the kind of repeated pairing with experience. Cognitive maps indeed do have that characteristic, if 
only because maps or even parts of maps are typically not uttered by the creature, as they are the hidden guiding structure. Of course maps, if real, are acquired on the basis of experience, and successful maze running indicative of maps is often rewarded. But we still do not have any expression of the map paired with a situation. Cognitive maps satisfy the first characteristic.

The second characteristic of symbols is that they are part of a system of symbols. Whether cognitive maps involve a system of symbols depends on what one takes a system to involve and what description of a map is thought to be appropriate. It could be said that there is some kind of system to a map as the nature of a map is the set of relationships among its significant parts, landmarks and so forth. This doesn't seem to be quite the notion of a system that Deacon is employing, though. He appears to be thinking of something based more on syntactic and semantic structure.

If we were to follow Gallistel's description of the computations involving the cognitive map then we are dealing with something involving that kind of complexity. The mathematical expressions he claims are evaluated have a recursive syntax and the property of compositionality. This makes them comparable to the expressions of a natural language from a syntactic point of view.

Gallistel's account of cognitive maps can be challenged if there are alternative, nonformalistic, ways of describing them. There seems to be no obvious bar to a connectionist system that produced the appropriate output without doing the mathematics. Certainly the analogy with the ordinary notion of a map does not suggest that formalistic treatment. The kind of map we use every day to help us find our way around is largely an analogue device. The arrangement of locations and relative distances between them is available to visual inspection, and more precise estimations of shortest routes can be found using that wonderful analogue device, a piece of string. While there is typically a grid to locate particular streets or cities, that can be regarded as additional feature to help identify particular locations in one's field of vision. If cognitive maps also have some kinds of analogue features, or in some way are capturable by a purely connectionist system, then they do not involve symbolic systems in the sense intended. 
The third characteristic concerns the basis of the symbols in a system of rich interactions with the environment characteristic of animals. Let us take it that the impressive navigational skills of the insects that Gallistel describes show that they meet this characteristic.

The fourth characteristic is that symbolic systems could support promises about the future. Leaving aside aspects of the speech act of promising such as intending and moral commitment that arguably are absurd to apply to the insect world we are left with the application of a proposition to the future. If a cognitive map could be said to express a proposition, which would depend on it involving structured expressions, then indeed it could be said to apply to the future. But, as we have found reason to doubt the need for those structured expressions, then we consequently have reason to doubt that a proposition about the future can be expressed.

The crucial point in this discussion is whether Gallistel is right to claim that dealing with a cognitive map involves manipulating mathematical expressions. While the matter is not settled, there is room for the view that insects with cognitive maps do not thereby have a symbolic system. What holds true for insects could well hold true for most or all non-human mammals. Moreover, the issue turns out not to depend on anything very peculiar to Deacon's notion of a symbol.

\subsection{Maybe neither insects nor humans manipulate symbols}

It appears we are not compelled to accept that symbol processing is ubiquitous in the animal world. But maybe we are not compelled to accept that symbol processing appears anywhere, which would be just as damaging for Deacon's position. We should look again at the way Sherman and Austin are said to have crossed the symbolic threshold. Could there be a connectionist explanation of their achievements, which avoids any appeal to cognitive symbols?

Connectionist systems can apparently perform certain kinds of generalizations without explicit representation of any general notions. Consider, for example, those that can 
produce the past tenses of regular and irregular English verbs without any explicit representation of the class of regular verbs (Rumelhart and McClelland 1986c). New cases of regular verbs can be handled without further training. There is no explicit representation of the past tense rule for regular verbs but the point most relevant here is that there is no explicit category of regular verb.

Connectionist systems get the benefits of a generalization by merely overlaying associations in a training sequence and acting on the basis of an implicit common pattern with no need for an explicit higher level category. Just as there is no explicit higher level category, so there is no registering of a relationship among each of the regular verbs that they are of the same kind. So there need be nothing like Sherman's and Austin's supposed recognition of relationships among lexigrams, if we were to model their achievements in a connectionist way. The simplicity of Sherman's and Austin's achievement makes the viability of a connectionist explanation seem especially plausible. Scaling up to human use of language with a full recursive syntax is more open to dispute, and there is considerable ongoing debate in, for example, Smolensky (1998), Fodor (1998b), and Fodor and McLaughlin (1998). On the face of it, Deacon needs to be able to rule out a connectionist explanation of both cases.

To deny, from a connectionist point of view, that humans or some other creatures manipulate symbols is likely to be the denial that there are distinct physical items that have a constant significance in different contexts. The focus is thus on the physical form of a symbol. But the requirement of constancy of significance links up with the second characteristic of symbols that I extracted from Deacon, which concerns the way symbols form a system. Deacon would appear to be committed to affirming the existence of symbols in humans in a way that is opposed to the connectionist approach.

A broadly connectionist approach is compatible with different views on representation, however. Gallistel (1998, p. 11) describes connectionism as abandoning any notion of representation, but his portrayal of the connectionist is an extreme one, which neglects connectionists' own professed intention to capture a notion of representation. "In our models we are explicitly concerned with the problem 
of internal representation and mental processing ..." (Rumelhart and McClelland, 1986b, p. 121).

There may be ways to integrate connectionism with Deacon's view. To pursue that possibility, we need to consider the extent to which Deacon is, or should be, a realist about symbols.

\section{How real are the symbol systems?}

The general tenor of Deacon's discussion of symbols, symbol systems and crossing the symbolic threshold suggests a fully realist approach to symbols, in the sense of holding that symbols are real items in the brain. Indeed, devoting a third of the book to the brain makes one anticipate an explanation of a clear neurological difference between a species such as ours that is equipped for symbol using and other species. But there are alternatives to a full realist approach to symbols.

\subsection{Instrumentalism and Holism}

In Deacon's account, the meaningfulness of symbols employed in thought depends on their membership of a system of symbols, which is a form of holism concerning their significance. In Dennett (1978) we see a good example of a kind of holism alongside a denial of realism, and we can usefully compare that with Deacon's approach to mental symbol systems. Dennett's holism concerns intentional states, that is, representational states such as beliefs and desires. His view is that something has intentional states just in case its behavior can be predicted successfully by attributing intentional states to it. This has been described as 'instrumentalism', supposing the attribution of the intentional states is a mere instrument in the predictive calculation. It portrays Dennett as not being realist about intentional states.

That apparent denial of realism sits comfortably with the holism. To attribute one belief to an individual we need to take the intentional stance towards it. We treat it as a rational agent and holistically construct a set of beliefs it ought to have given its contact with the world, along with a set of desires it ought to have given its 
environmental niche (Dennett 1987b, p. 17). This enables us to provide predictions of the individual's behavior without supposing that there is something in the individual that corresponds to each attributed belief and desire, let alone any word-like components of those intentional states. In his later work, Dennett (1987b, 1987c and 1998) retreats from the earlier appearance of a complete denial of realism and claims to adopt a sort of realism, which is a view we will return to.

\subsection{Deacon's realism and holism}

As explained above, Deacon thinks that the meaning and reference of symbols in the mind of a human being is not determined individually but holistically. The symbol system as a whole connects with the observable world; the connection is not made piecemeal, symbol by symbol. The general tone of Deacon's view of symbols is realist, though. The very talk of 'the symbolic species' suggests realism and the material on the neurological basis of the human symbol system reinforces that interpretation.

While instrumentalism of the kind attributed to the early Dennett requires holism, it is not obvious that holism requires instrumentalism. One can hold that a perfectly real set of symbols must be mutually self-supporting, like the poles of a frame tent. Deacon could hold that a perfectly real system of symbols both underpins the meaningfulness of a person's utterances and provides a basis for their intentional states. There needs to be caution in attributing symbol realism to Deacon, though, and also it leads to the symbolic threshold being a sharp threshold, which is problematic, as we shall see.

Deacon's account is that symbols are distributed through various parts of the brain. It seems natural to say that the symbolic system is a virtual system supported by a connectionist style of neural computation. Deacon (p. 265) himself speaks of 'implicit symbolic reference' and the way it 'emerges from a pattern of virtual links' (p. 266). He also appears to endorse the work of Elman (1993) in using connectionist systems to learn complex syntactic structure (Deacon, 1997, p. $132 \mathrm{ff}$ ). 
Merely describing Deacon's symbol systems as 'virtual' does not settle the matter of their reality, for the notion of a virtual machine is compatible with different levels of realism. One can specify a virtual machine in relation to a more basic machine. A piece of application software creates a virtual machine where the base machine is a computer, with its operating system. But the operating system itself, which may be largely a software construct, can itself be regarded as a virtual machine. Some hardware description may specify a base machine but the division of function between hardware and software is to some extent arbitrary. It is best to say that pieces of software create functioning systems that are fully real.

In biological systems the distinction between what is innate and what is acquired can be seen as parallel to the hardware-software division. But, again, the line is not as clear as it first seems, as the DNA code contributes to form only in a certain environment (Deacon, 1997, Chapter 7; Dennett, 1995, pp. 113-115). In considering the place of symbols in the brain in Deacon's system, it seems natural to say that the system of symbols is created in the brain, relying on the basic plasticity of the brain and on the symbol creating tendencies that are inherited. We shouldn't deny reality to a mental system just because it is learned. There is a parallel with Dennett's view of the way that consciousness depends on a virtual von Neumann machine realized on the connectionist base of the brain (Dennett 1991, p. 218).

Nonetheless, there may be reason to question the reality of the virtual symbol system. Even if a connectionist system, for the most part, is an implementation of a high-level structure, its behavior may only approximate the high level description. Interest in connectionist models can lie in relatively small divergences from the high level descriptions (McClelland et al., 1986, p. 12). Holding that the symbols are distributed in the brain makes it more likely that the underlying brain structure diverges a little from the description at the symbolic level.

Rumelhart, et al. (1986, especially p. $44 \mathrm{ff}$ ) have an approach to the creation of such a virtual symbolic machine in which what the brain does is initially model external symbols and then manipulate them purely internally. Andy Clark (1990, Chapter 7) 
incorporates discussion of this passage into his exploration of the possibility that there is no unitary architecture for the mind. The fundamentally connectionist architecture of the brain can support a symbol processor, which is the basis of some of our cognitive processes but not all. While Rumelhart et al. (1986) only speak of the construction of the internal symbol processor happening in one individual, Clark (1990, p. 134) speculates that the modification could become part of our innate brain structure as a result of natural selection, though only as kind of kludge. This gives us a way of understanding what the brain adaptation might amount to in Deacon's coevolutionary process.

One of the issues that remain is whether the description of the virtual symbol processor could ever be more than an approximation of the underlying brain behavior. The connectionist line is that the connectionist basis of the symbol system is more than an implementation detail and so the description of the symbol system is never more than an approximation. If this were the correct way to regard Deacon's symbols, there would be reason to qualify our initial realist interpretation of his view. Deacon's text does not provide clear support for that interpretation, though.

We need not go to the other extreme and conclude that the high level description is largely fictional or instrumentalist. In fact the best approach may be to adopt the form of 'mild realism' that Dennett adopts in later work. He speaks of there being objective patterns in human affairs that can be only detected from the intentional stance (Dennett 1987b, p. 25). Patterns, as Dennett (1998, p. 99ff) explains them, can be imperfectly instantiated, for the signal, which is the pure pattern, can be partially distorted by noise.

Maybe we should also say there are real patterns in the brain, the symbols, that can similarly only be detected using a symbolic stance (and maybe, in the future, a connectionist, symbol-detecting, brain scanner). Even if the connectionist neural system corresponds only imperfectly to the symbolic system, this can be accepted as the signal being somewhat distorted by noise. In fact the symbolic stance to the brain would be closely related to, and have to depend ultimately on, the intentional stance to 
the whole person, as we could only get any purchase on a symbolic system by interpreting some behavior in an intentional way.

A parallel is here being drawn between Dennett's mild realism about intentional states and a proposed mild realism about symbols in the brain. Incidentally Dennett, for a long time, has recognized that language use has enhanced our ability to have beliefs. He has made a distinction between belief and opinion (Dennett, 1978c), where an 'opinion' is a belief that the believer can formulate to herself in words. Dennett is happy to attribute beliefs where there are no opinions, but his interest in opinions suggests an openness to there being some sort of threshold associated with language use.

The outcome of this consideration of Deacon's notion of a symbol is that, while his holism appears to accompany a form of realism about symbols, it may be wise to entertain a mild realist position about them. Deacon's position can be reinterpreted to be compatible with Dennett's position about real patterns. Even if this can be contested as an accurate exegesis of Deacon's position it is a position that has much to recommend it. There is something extremely implausible about a full realist approach to symbols in the brain, as that goes hand in hand with a sharp threshold.

Consider the point at which Sherman and Austin have supposedly crossed the symbolic threshold. They had already been trained to associate individual lexigrams with items, but in Deacon's view they were not employing them as symbols. For Deacon, crossing the threshold is the moment when they recode the signs they know to relate them to each other. Suddenly we have the miracle of word meaning and reference. Deacon (p. 93) is perfectly explicit that the threshold at an individual level is perfectly sharp, as it is the moment of perceiving the relationship amongst signs, as described in section 2.2. Moreover, within a group, one individual must have crossed that threshold first at the very beginnings of language. Of course, there are likely to have been many false starts, as Deacon allows (p. 402). The problem is, how could anyone have crossed the threshold without language in the environment? In speaking of language we should not just think of spoken language as we are talking of a time 
before the descent of the larynx. Deacon (p. 407) describes a plausible mixture of vocalizations, gestures and use of objects.

The problem of how to cross the threshold without an existing language is especially clear if we consider the suggestion in Rumelhart et al. (1986) that internal symbols were initially models of external symbols. But even without the assumptions involved in that approach the question remains. We seem to need to have a 'language' or at least some kind of external symbol system prior to any individual crossing the symbolic threshold internally. On Deacon's view, though, words of that hypothetical language would have lacked meaning, for crossing the symbolic threshold is what produces word meaning. We seem to require a structure of calls or signs that, while lacking meaning in itself, could be interpreted by an innovating individual to be composed of meaningful inter-related symbols.

When Deacon discusses the origins of symbols in rituals he may be attempting to describe such a situation. He describes the Yanomamö peace rituals (pp. 403-5) in which there are series of threats that are not responded to. He also describes marriage rituals (p. 406). For rituals to serve the role of pre-symbolic systems that lead up to the crossing of the symbolic threshold we need to suppose that they existed prior to the existence of even simple language. Even if rituals did play a role in a cognitive shift in early language development it seems extreme to require that their emergence strictly pre-dated the beginnings of deliberate communicative language. Indeed the position is hard to understand even on Deacon's terms. To have a ritual is to have a system of signs, not just a series of isolated signs. Once there is a system we have the central requirement of being a symbol system. If the ritual is a symbol system, we are brought back to the question, how can individuals who lack symbolic thought create a symbol system?

\subsection{Mild Realism about Symbols}

The apparent sharpness in Deacon's conception of the threshold stems from the combination of holism and realism. Symbol realism without the holism could permit a gradualistic conception of the threshold, or even no threshold at all, for it could be 
thought that symbols appear one by one. It is the notion that symbols must intrinsically be part of a symbol system that rules out that possibility. Holism without realism also permits a fuzzy threshold or no threshold at all, as we shall see. It is the combination of holism and realism that causes the troublesome sharpness of the threshold, which makes it very hard to describe the lead up phase and the actual transition.

While a perfectly sharp threshold appears to be a conceptual roadblock, this is a separate matter from the debate concerning the speed of language emergence. Jean Aitchison (1998), for example, distinguishes the 'slow haul' hypothesis from the 'pop' hypothesis in which languages 'pop' into existence. Leaving aside the conceptual requirements of a sharp threshold there may well be reasons for thinking that the process must have been fast. A group maybe needs to be able to advance quickly together in order for the individuals to provide for each other the linguistic environment that leads them into a new way of thinking and behaving. That claim could still be made even without the artificial requirement of a perfectly sharp threshold

Dennett, interestingly, combines holism about intentional states with gradualism. He denies that there is a 'magic moment' along the scale of ever more complex organisms at which they really have internal representations (Dennett 1987b, p. 32). He can combine holism and gradualism because he has adopted no more than mild realism. He can describe different degrees of intentionality as cases of a signal being distorted by different amounts of noise. Clark $(1990,4.4)$ also employs a notion of gradualistic holism, but it is not precisely the notion we need here. It concerns the way a complex whole is built out of parts that have evolved separately as complete units.

Let us pursue a similar mild realist interpretation to symbols. Where Dennett talks primarily about patterns in human behavior we should here talk of patterns of brain activity. Symbols can be regarded as virtual symbols realized in that connectionist base, as explained above in connection with Rumelhart et al. (1986) and Clark (1990). There is scope on this interpretation for there to be brain states that express symbols to different degrees, where the signal is distorted by different amounts of noise. If this 
thinking is combined with Deacon's emphasis on a symbolic threshold we could say there were a series of states of the brain going over the threshold that express the pattern of a symbolic system with progressively less noise. It would allow him to describe some intermediate cases as ones in which there was scarcely a mental symbol system.

If a mental symbol system's existence can be a matter of degree, then the existence of word meaning and reference can also be a matter of degree. If there are degrees of reality of word meaning and reference, we can say some series of sounds, gestures or marks form what is scarcely or marginally a language. If we relax the realism assumption about symbols it may also be natural to relax the holism assumption. While may the development of complex messages may have been important in bringing about the cognitive shift it would be sensible to allow that single sign messages for such things as "Look over there!" or "Return home!" could have been at least marginally symbols. This by no means solves all the real and troublesome questions about how language began, but at least the concepts permit the description of crucial intermediate stages that are required of a plausible explanation.

Deacon solved the problem of how one puts together a system without any component parts by insisting on a firm division between indices and symbols and allowing that indices but not symbols can be learned individually. The indices are the parts that can be converted into symbols at one fell swoop. Deacon's use of Peirce's terms 'index' and 'symbol' has already been questioned in section 2.1. His rigid division between 'index' and 'symbol' is an essential part of an approach that involves a sharp threshold. The consequence, as we have seen, is an implausible reliance on rituals preceding languages.

Any detailed account of the crossing of the threshold, making use of a terminology that allows some intermediate stages, can still allow that the threshold is a barrier. Crossing the barrier may be difficult because of the costs of the cognitive reorganization and it may need to be fast. Those things are both consistent with the need for some intermediate stages. Deacon can still explain why there are no simple languages in other species, on the basis of the difficulty of crossing the threshold. 
Thus a mild realist interpretation of symbols permits the expression of some of the central features of Deacon's position without a troublesome sharp threshold.

\section{Acknowledgments}

Thanks to the philosophers at the University of Hull for their hospitality in autumn 1999. Thanks also for the comments of participants at seminar presentations of earlier versions at the Universities of Waikato, Glasgow, Stirling, Hull and York. Thanks also to Norman Melchert and two anonymous reviewers.

\section{Bibliography}

Aitchison, J. (1998). On discontinuing the continuity-discontinuity debate. In Hurford et al., 17-29.

Bates, E. (1990). Language about Me and You: Pronominal Reference and the Emerging Concept of Self. In Cicchetti, D. and Beeghly, M. (Eds.) The Self in Transition. Chicago: University of Chicago Press, 165-82.

Buchler, J. (Ed.) (1955). Writings of Peirce. New York: Dover.

Chomsky, N. (1988). Language and Problems of Knowledge: The Managua Lectures. Cambridge MA: MIT Press.

Clark, A. (1990). Microcognition: Philosophy, Cognitive Science, and Parallel Distributed Processing. Cambridge MA: MIT Press.

Deacon, T.W. (1997). The Symbolic Species: The Co-evolution of Language and the Brain. New York: Norton.

Dennett, D. (1978a). Brainstorms. Cambridge MA: MIT Press. (1978b). Intentional Systems. In Dennett 1978a, 3-22. (1978c). "How to Change Your Mind," in Dennett 1978a, 300-309. (1987a). The Intentional Stance, Cambridge MA: MIT Press (1987b). True Believers: The Intentional Strategy and Why It Works. In Dennett 1987a, 13-35.

(1987c). Reflections: Real Patterns, Deeper Facts, and Empty Questions. In Dennett 1987a, 37-42. (1991). Consciousness Explained. Boston: Little, Brown \& Co,. (1995). Darwin's Dangerous Idea. New York: Simon and Schuster. (1998). Real Patterns. In Brainchildren. Essays on Designing Minds. London: Penguin Books, and Cambridge MA: MIT Press

Elman, J. (1993). Learning and Development in Neural Networks. The Importance of Starting Small. Cognition, 48, 443-448. 
Fodor, J.A. (1998a). In Critical Condition. Polemical Essays on Cognitive Science and the Philosophy of Mind. Cambridge MA: MIT Press

(1998b) Connectionism and the Problem of Systematicity (Continued): Why Smolensky's Solution Still Doesn’t Work. In Fodor 1998a, 113-125.

Fodor, J.A. and B. McLaughlin (1998). Connectionism and the Problem of Systematicity: Why Smolensky's Solution Doesn't Work. In Fodor 1998a, 91111.

Gallistel, C.R. (1998). Symbolic Processes in the Insect Brain. In Scarborough and Sternberg, 1-51.

Hookway, C. (1985). Peirce. London: Routledge \& Kegan Paul.

Hurford, J.R., M. Studdert-Kennedy and C. Knight (1998). Approaches to the Evolution of Language. Cambridge, U.K.: Cambridge University Press.

Kaplan, D. (1989). Demonstratives. In J. Almog, J. Perry and H. Wettstein (Eds), Themes From Kaplan. New York: Oxford University Press.

Lewontin, R.C. (1998). The Evolution of Cognition: Questions We Will Never Answer. In Scarborough and Sternberg.

McClelland, J.L., D.E. Rumelhart, and G.E. Hinton (1986). The Appeal of Parallel Distributed Processing. In Rumelhart and McClelland, 1986a Vol. 1, 3-44.

Rumelhart, D.E., and J.L. McClelland (1986a). Parallel Distributed Processing: Explorations in the Microstructure of Cognition, Cambridge MA: MIT Press. (1986b). PDP Models and General Issues in Cognitive Science. In Rumelhart and McClelland, 1986a Vol. 1, 110- 146. (1986c). On Learning the Past Tenses of English Verbs. In Rumelhart and McClelland, 1986a Vol. 2, 216-271.

Rumelhart, D. E., P. Smolensky, J.L. McClelland and G.E. Hinton, (1986). Schemata and Sequential Thought Processes in PDP Models. In Rumelhart and Mclelland, 1986a Vol. 2, 7 - 57.

Savage-Rumbaugh, E. S., D.M.Rumbaugh, and S. Boysen (1978). Symbolization, Language and Chimpanzees: A theoretical reevaluation based on initial language acquisition processes in four young Pan troglodytes. Brain and Language 6, 265.

Scarborough, D. and Sternberg, S. (1998). An Invitation to Cognitive Science, Volume 4, Methods, Models and Conceptual Issues. Cambridge, MA: MIT Press.

Smolensky, P. (1988). On the Proper Treatment of Connectionism. Behavioral and Brain Sciences 11, 1-23. 